\title{
Durability of left ventricular assist devices: Interagency Registry for Mechanically Assisted Circulatory Support (INTERMACS) 2006 to 2011
}

\author{
William L. Holman, MD, ${ }^{\text {a }}$ David C. Naftel, PhD, ${ }^{a}$ Chad E. Eckert, PhD, ${ }^{b}$ Robert L. Kormos, MD, \\ Daniel J. Goldstein, MD, ${ }^{\mathrm{c}}$ and James K. Kirklin, $\mathrm{MD}^{\mathrm{a}}$
}

Objectives: The present study compared the interval until device exchange or death from pump-related failure in patients with pulsatile versus continuous flow left ventricular assist devices.

Methods: Data from Interagency Registry for Mechanically Assisted Circulatory Support (June 23, 2006, to March 31,2011) compared the durability of implanted pulsatile and continuous flow left ventricular assist devices. The durability issues included pump replacement for infection, thrombosis-hemolysis, driveline failure, or pump drive unit failure, and death from driveline or pump drive unit failure.

Results: A total of 3302 left ventricular assist devices were implanted (486 pulsatile, 2816 continuous flow) and 98 pump exchanges or deaths from durability issues (46 pulsatile, 52 continuous flow; $3 \%$ of implants). The interval to device issue was greater for the continuous flow than for the pulsatile devices $(P<.001)$. A comparisons of the causes for pump exchange or pump-related death showed (1) greater freedom from pump failure in the continuous flow compared with the pulsatile left ventricular assist devices ( 10 events/2816 continuous flow implants vs 39 events/486 pulsatile implants; $P<.0001)$; (2) similar exchange or pump-related death for driveline failure (1/486 pulsatile vs $7 / 2816$ continuous flow; $P=.82$ ); (3) similar exchange or pump-related death for thrombosis-hemolysis ( $2 / 486$ pulsatile vs $28 / 2816$ continuous flow; $P=.25$ ); and (4) fewer exchanges or pumprelated deaths from infection in continuous flow left ventricular assist devices (4/486 pulsatile vs 7/2816 continuous flow; $P=.034)$. Competing outcomes analysis corroborated this finding, with $54 \%$ of continuous flow versus $23 \%$ of pulsatile patients alive and receiving support at 12 months after implantation.

Conclusions: The Analysis of Interagency Registry for Mechanically Assisted Circulatory Support data showed greater durability for continuous flow than for pulsatile left ventricular assist devices. Even longer durations of support can be expected if pump durability continues to improve. (J Thorac Cardiovasc Surg 2013;146:437-41)

\section{Supplemental material is available online.}

Patients receiving a mechanical circulatory support device (MCSD) place their lives in the hands of the engineers who design the MCSDs and the physicians who manage the MCSDs. From the patient's standpoint, the ideal MCSD would have sufficient durability to sustain their life until they die of something other than heart failure, mechanical pump failure, or a complication of MCSD therapy.

\footnotetext{
From the Department of Surgery, ${ }^{\text {a }}$ University of Alabama at Birmingham, Birmingham, Ala; Departments of Bioengineering and Surgery, ${ }^{\mathrm{b}}$ University of Pittsburgh, Pittsburgh, Pa; and Department of Cardiothoracic Surgery, ${ }^{c}$ Montefiore-Einstein Medical Center, Bronx, NY.

Disclosures: Authors have nothing to disclose with regard to commercial support.

Received for publication Oct 24, 2012; accepted for publication Feb 12, 2013; available ahead of print March 13, 2013.

Address for reprints: William L. Holman, MD, Department of Surgery, University of Alabama at Birmingham, Room 719, 703 19th St S, Birmingham, AL 35294-0007 (E-mail: wholman@uab.edu).

0022-5223/\$0.00

Published by Elsevier Inc. on behalf of The American Association for Thoracic Surgery

http://dx.doi.org/10.1016/j.jtcvs.2013.02.018
}

From an engineering and clinical perspective, the reliability or durability is measured by the rate of device failure over time. ${ }^{1}$ MCSD failure can be the result of a mechanical problem (eg, drive unit or motor failure of an implanted ventricular assist device [VAD]) or a biologic complication involving the MCSD (eg, device thrombosis or hemolysis). The most devastating failures of durability result in death of the patient. If MCSD failure is not fatal but requires replacement of the implanted components, it exposes the patient to the important risks of death and other adverse events.

In the present study, we analyzed the durability of MCSDs that have been approved by the Federal Food and Drug Administration (FDA) and tracked by the Interagency Registry for Mechanically Assisted Circulatory Support (INTERMACS). ${ }^{2}$ Our aim was to define the MCSD failure rates for several modes of failure and to compare the failure rates and modes of failure for the 2 major classes of MCSDs: pulsatile and continuous flow (CF) pumps. The ultimate goals were to (1) stimulate improvements in the engineering of MCSDs; and (2) suggest changes in the clinical treatment of patients to maximize MCSD durability. 


\section{Abbreviations and Acronyms \\ $\mathrm{CF} \quad=$ continuous flow \\ FDA $\quad=$ Food and Drug Administration \\ INTERMACS $=$ Interagency Registry for \\ Mechanically Assisted Circulatory \\ Support \\ LVAD $\quad=$ left ventricular assist device \\ MCSD = mechanical circulatory support device \\ $\mathrm{VAD}=$ ventricular assist device}

\section{METHODS}

The INTERMACS is a national registry for patients who receive a MCSD to treat advanced medically refractory heart failure. The INTERMACS indications for mechanical circulatory support include destination therapy (ie, a long-term MCSD for patients with a contraindication to transplantation), bridge to transplantation, and bridge to recovery of the native heart. Only FDA-approved MCSDs that can be used in an outpatient setting are included in the INTERMACS. ${ }^{2}$

A total of 106 institutions were enrolled in the INTERMACS from June 23, 2006 to March 31, 2011, the period of data acquisition for the present study. The data were entered using encryption to a secure server administered by the United Network for Organ Sharing. The United Network for Organ Sharing data repository is accessible to INTERMACS investigators for analysis of patient de-identified information. The study sites and central data processing and analyzing facilities received institutional review board approval before initiating the collection of data. All patients provided informed consent before entry in the INTERMACS. The data were managed according to the Health Insurance Portability and Accountability Act regulations.

During this phase of data acquisition, the MCSDs included in the INTERMACS database included the AbioCor total artificial heart (Abiomed, Danvers, Mass); the HeartMate IP, VE, and XVE left VADs (LVADs; Thoratec, Pleasanton, Calif); the HeartMate II LVAD (Thoratec), the MicroMed DeBakey Child LVAD (MicroMed, Houston, Tex); the Novacor PC and PCq LVADs (Novacor, Oakland, Calif); the SynCardia Cardiowest total artificial heart (Syncardia Systems, Tucson, Ariz); and the Thoratec implantable VAD and paracorporeal VAD pumps (Thoratec, Pleasanton, Calif). ${ }^{3}$ The total artificial hearts and paracorporeal VADs were not included in the present analysis. Only adults aged 18 years or older were included in the present study; thus, the MicroMed DeBakey Child LVADs were excluded.

The INTERMACS variables describing the preimplant demographic profile, indication for mechanical circulatory support, and clinical variables that described the preimplant patient condition, hemodynamics, and laboratory values were analyzed to define the risk factors for postoperative device durability. The relevant durability issues included pump replacement for intractable infection, thrombosis-hemolysis, driveline failure, or pump drive unit failure and death from driveline or pump drive unit failure. The patients were censored from the analysis because of transplantation, device removal for recovery, and death not related to pump failure.

The INTERMACS data were checked for completeness by the central collection facility (United Network for Organ Sharing). The data that were outside the predetermined limits were validated by their site of origin; however, the source documents were not routinely checked against the data submitted to the INTERMACS. The adverse event forms were reviewed by physician volunteers from the INTERMACS community and checked further by the members of the INTERMACS adverse event committee before a final adjudication was made on the individual adverse events.
The variables describing the preimplant patient condition, indication for mechanical circulatory support, demographic profile, hemodynamics, laboratory values, and durability issues were gathered. The data were analyzed using a competing outcomes method to identify the risk factors for postoperative pump exchange and death from durability issues. The pulsatile and CF rotary pumps were compared for durability as an aggregate outcome and were compared again according to the individual durability issues specified in the previous paragraphs.

\section{RESULTS}

The data for 3302 patients were entered by 106 institutions within the study period, with a follow-up date of March 31, 2011. Pulsatile pumps were approved for clinical use by the Federal FDA before CF pumps; therefore, these 2 groups were nonconcurrent. Of the 3302 LVADs implanted, 486 were pulsatile and 2816 were CF LVADs. The total experience included 2624 patient-years of support and 585 nonpump-related deaths. Also, 100 pump exchanges occurred and 11 deaths from durability issues (59 pulsatile deaths/exchanges and $52 \mathrm{CF}$ deaths/exchanges; Table 1). The pumprelated issues that led to exchange or death are listed in Table $\mathrm{E} 1$, and the interval to device issue (pump exchange or pump-related death) is shown in Figure 1. The CF devices failed predominantly because of pump thrombosis and hemolysis (28/52 [54\% of events]), and the pulsatile pump failed predominantly because of pump drive unit failure (46/59 [78\% of events]). The interval to all-cause pump-related failure (death or pump exchange) was significantly $(P<.0001)$ longer for CF pumps than for pulsatile pumps.

The causes for durability failure are depicted individually in Figures 2, 3, E1, and E2. The durability, as defined by drive unit failure, was significantly better for the CF pumps than the pulsatile pumps $(P<.0001$; Figure 2$)$. The interval to the first driveline problem, thrombosis-hemolysis, or infection that required device exchange was similar for the pulsatile and CF LVADs (Figures 3, E1, and E2). The competing outcomes analyses for pulsatile and CF pumps (Figure 4) corroborate the greater durability of CF pumps compared with pulsatile pumps. This was evidenced by the greater proportion of CF patients remaining on pump support at 12 months after device implant $(54 \%$ of 2816 CF implants vs $32 \%$ of 486 pulsatile LVADs), although other possible explanations exist for the finding. Other explanations include a greater proportion of patients with panel reactive antibodies or blood group $\mathrm{O}$ or a greater number of destination therapy patients than bridge to transplantation patients in the CF group.

\section{DISCUSSION}

The concept of durability is of obvious importance to cardiac replacement therapies. In 1998, a joint American Society for Artificial Internal Organs-Society of Thoracic Surgeons committee published recommendations and goals for the development and clinical evaluation of MCSDs. ${ }^{4}$ That document defined failure as "the termination of the 
TABLE 1. Reasons for pump exchange or device-related problem that contributed to patient death stratified by pump type (pulsatile vs continuous flow)

\begin{tabular}{lcc}
\hline & \multicolumn{2}{c}{ Pump type } \\
\cline { 2 - 3 } \multicolumn{1}{c}{ Outcome } & Continuous flow & $\begin{array}{c}\text { Pulsatile } \\
\text { intracorporeal flow }\end{array}$ \\
\hline Dead & $424(15.06)$ & $171(35.19)$ \\
$\quad$ Non-pump related & $420(14.91)$ & $164(33.74)$ \\
Pump related & & \\
$\quad$ Thrombosis/hemolysis & $3(0.11)$ & $2(0.41)$ \\
$\quad$ Drive line & $1(0.04)$ & $0(0.00)$ \\
$\quad$ Drive unit & $0(0.00)$ & $5(1.03)$ \\
Transplant & $809(28.73)$ & $247(50.82)$ \\
Recovery & $19(0.67)$ & $12(2.47)$ \\
Device exchange & $48(1.70)$ & $52(10.70)$ \\
Infection & $7(0.25)$ & $4(0.82)$ \\
Pump related & & \\
$\quad$ Thrombosis/hemolysis & $25(0.89)$ & $6(1.23)$ \\
$\quad$ Drive line & $6(0.21)$ & $1(0.21)$ \\
$\quad$ Drive unit & $10(0.35)$ & $41(8.44)$ \\
Alive & $1516(53.84)$ & $4(0.82)$ \\
Total & $2816(100)$ & $486(100)$ \\
\hline
\end{tabular}

Data presented as $\mathrm{n}(\%)$.

ability of an item to perform a required function or the inability of the device or any of its components to meet performance specifications." 4 The committee also suggested metrics for reliability based on in vitro testing (eg, mock circulation loop), animal device implants, and clinical trials (eg, investigational device exemption trials). The present analysis had a somewhat different perspective on device failure because we only used data gathered from the postmarketing surveillance of FDA-approved MCSDs ${ }^{5}$ and included modes of failure that might not be discovered in animal trials (eg, device exchange because of intractable

Time to Device Issue (Exchanged or Contributed to Death)

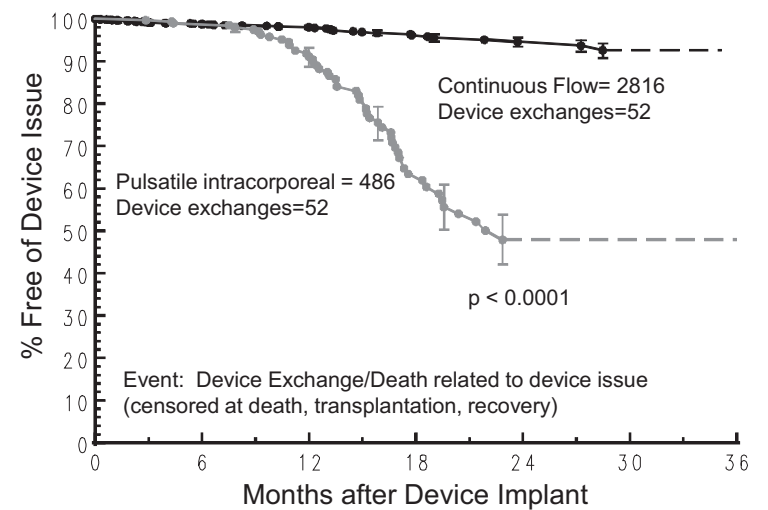

FIGURE 1. The intervals to device exchange or device-related death for all causes are depicted for pulsatile and continuous flow ventricular assist devices $(P<.0001$; pulsatile vs continuous flow ventricular assist devices).

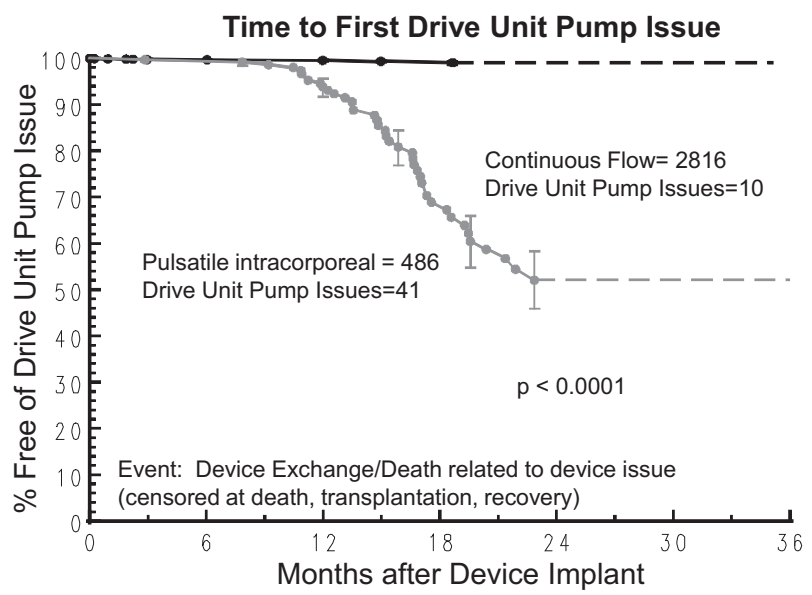

FIGURE 2. Deaths and device exchanges from drive unit failure are shown for pulsatile and continuous flow ventricular assist devices $(P<.0001$; pulsatile vs continuous flow ventricular assist devices).

device-related infection). These postmarketing surveillance data were gathered by the INTERMACS, a collaborative effort of physicians, industry, the National Institutes of Health, the FDA, and the Centers for Medicare and Medicaid Services. ${ }^{6,7}$

The INTERMACS captures the vast majority of LVAD implants in North America. The data entered at the individual sites are checked by the INTERMACS, although not to the extent of an investigational device exemption trial. The INTERMACS does not include data from MCSDs that are in clinical trials. Therefore, no centrifugal design rotary pump (eg, HeartWare LVAD; HeartWare International, Framingham, Mass) has been represented in the INTERMACS. Despite these limitations, the INTERMACS provides the

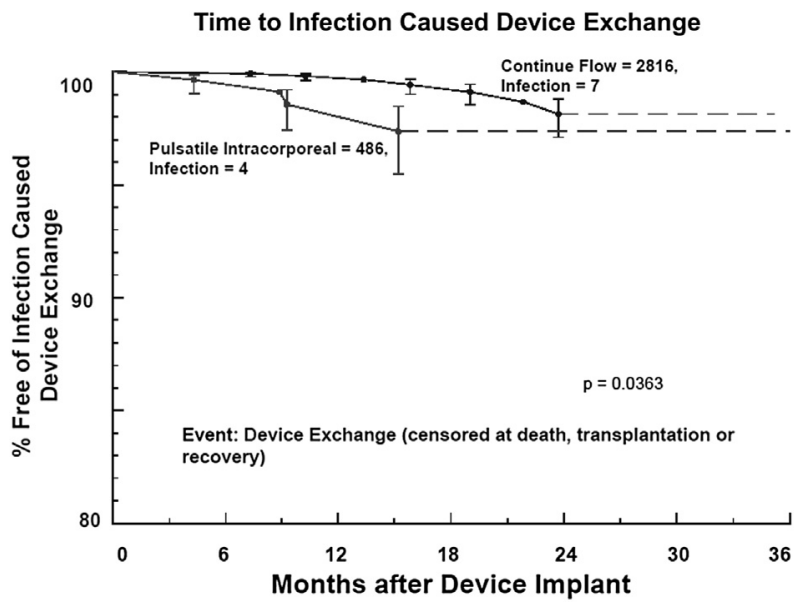

FIGURE 3. Interval to device exchange or patient death from intractable device-related infection is depicted for pulsatile and continuous flow ventricular assist devices $(P<.0363$; pulsatile vs continuous flow ventricular assist devices). 

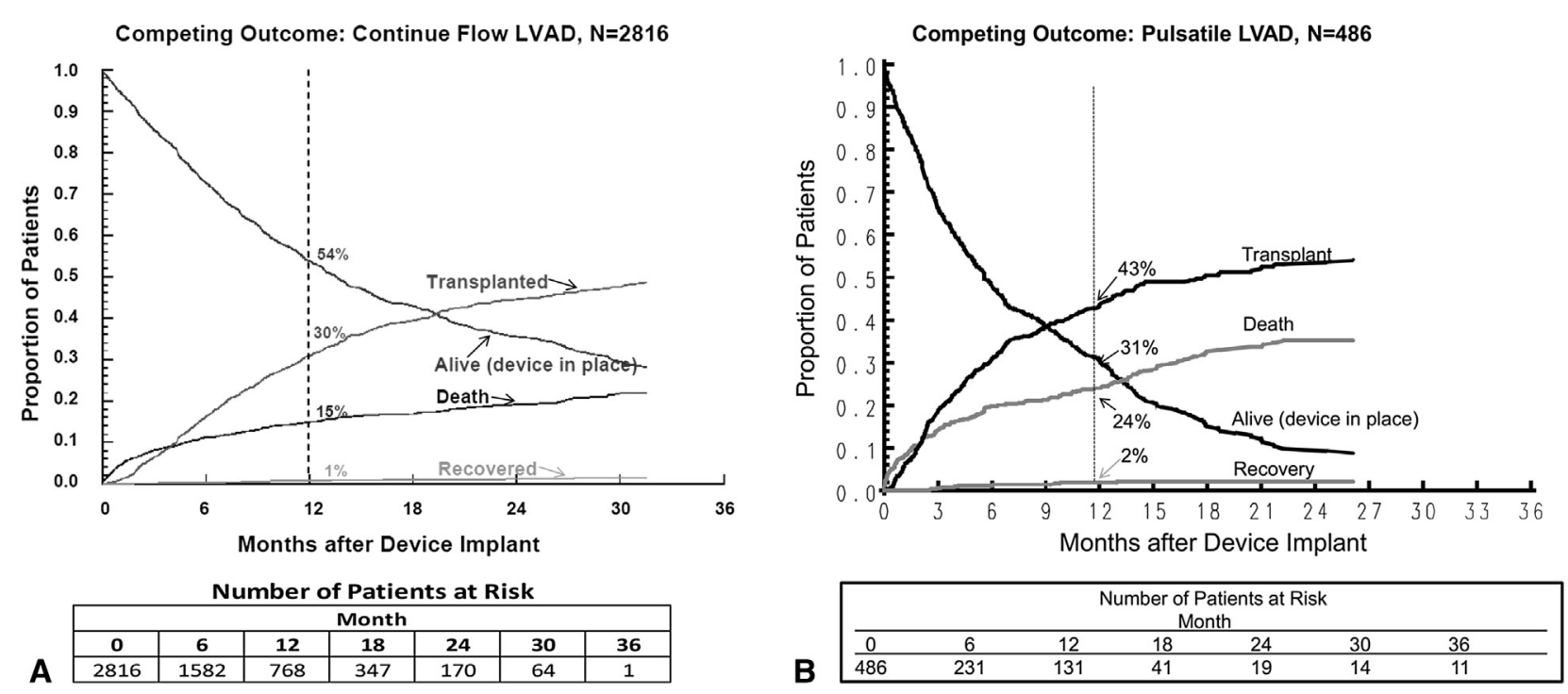

FIGURE 4. A, Competing outcomes (cardiac transplantation, death, survival on mechanical circulatory support device, and recovery of the native heart) for patients with continuous flow ventricular assist devices. B, Competing outcomes (cardiac transplantation, death, survival on mechanical circulatory support device, and recovery of the native heart) for patients with pulsatile ventricular assist devices.

most robust information currently available on the outcomes of patients supported by MCSDs. ${ }^{5,8}$

Device durability is extremely important to MCSD patients and their physicians. Device failure for any reason typically requires pump replacement, which adds substantial cost to MCSD therapy, exposes the patient to possible death or morbid events, and diminishes the patient's quality of life. The present report was designed to measure the failure rates for implanted LVADs and to compare these rates between pulsatile and CF pumps.

Pulsatile LVADs were represented in the INTERMACS predominantly by the pulsatile HeartMate I (Thoratec). Moreover, the Randomized Evaluation of Mechanical Assistance for the Treatment of Congestive Heart Failure (REMATCH) trial used the HeartMate I for the MCSD arm of the study, which compared survival and other outcomes for patients with end-stage heart disease treated with optimal medical management versus mechanical circulatory support using a pump approved by the FDA. ${ }^{9}$ That landmark trial demonstrated the superiority of mechanical circulatory support over medical management in patients with severe heart failure; however, it also illustrated the drawbacks of mechanical circulatory support. These included substantial postimplant mortality for LVAD patients at $1(48 \%)$ and 2 $(77 \%)$ years and a high rate of pump replacement for pump failure 12 to 18 months after implantation.

The Investigation of Nontransplant-Eligible Patients Who Are Inotrope Dependent (INTrEPID) trial compared the pulsatile Novacor LVAD (WorldHeart, Oakland, Calif) and optimal medical therapy for inotrope-dependent patients with New York Heart Association functional class IV symptoms. The INTrEPID trial was a nonrandomized study that corroborated the superiority of MCSD over medical therapy using a pulsatile LVAD. ${ }^{10}$ The patients in the LVAD arm of that study had a $27 \%$ 12-month survival, and mechanical failure of the Novacor LVAD was not listed as a cause of death or an adverse event for any patient. In the present trial, cerebrovascular accidents (10/37 patients), infection (7/37 patients), and cardiac dysfunction (5/37 patients) were the most commonly reported causes of death.

The results of clinical trials stimulated design changes in pulsatile pumps that resulted in improved patient outcomes, defined as improved survival with fewer adverse events. ${ }^{11,12}$ However, the disadvantages of pulsatile implantable blood pumps compared with implanted rotary CF pumps (eg, patient acceptance, ease of implantation) resulted in dominance of the field by CF designs. From the data from the present study, the durability of CF pumps compared with pulsatile pumps is an important aspect of the CF pump advantage. The importance of improved durability was noted in the first FDA investigational device exemption trial that compared an axial flow rotary pump to a pulsatile design. ${ }^{13}$ The INTERMACS data were used in the present analysis to quantify the improved durability of rotary pumps from experience at multiple centers.

Despite the improved durability of CF LVADs, the present analysis identified problems with the CF designs that led to pump failure, defined as pump-related adverse events causing patient death or requiring pump exchange. Pump thrombosis and pump-related hemolysis were identified in 28 of 52 of these instances $(54 \%)$, and intractable infection, drive unit failure, and driveline problems were each responsible in $13 \%$ to $19 \%$ of instances. Importantly, all 4 of these problems are amenable to improvement through design 
optimization by engineers (eg, improved hemocompatibility of blood contacting materials; diminished turbulence, and shear forces within the pump) or changes in patient treatment (eg, patient-specific anticoagulation ${ }^{14,15}$; novel methods for driveline implantation and stabilization; implementation of an intensive surveillance protocol for out-patients ${ }^{16}$ ).

The clinical results from previous multicenter randomized $^{17}$ and nonrandomized ${ }^{18}$ studies corroborate the findings of the present analysis regarding the improved durability of CF LVADs compared with pulsatile LVADs. In a 2009 study by Pagani and colleagues, ${ }^{18}$ the 18 -month actuarial survival for the HeartMate II LVAD recipients was $72 \%$ (95\% confidence interval, 65\%-79\%), and in a 2009 study by Slaughter and colleagues, ${ }^{19}$ the 24 -month actuarial survival for the HeartMate II LVAD recipients was $58 \%$ (95\% confidence interval, 49\%-67\%).

As investigators identify the features of LVADs that limit their useful life, subsequent improvements can be expected that will increase pump durability and patient survival. With these improvements, it can be expected that survival will become equivalent between LVAD therapy and heart transplantation for certain patient groups with advanced heart failure. ${ }^{19}$ Furthermore, MCSDs might be routinely used before and after heart transplantation to maximize patient survival through sequential MCSD and transplantation therapies.

\section{References}

1. Fries RC. The concept of failure. In: Reliable Design of Medical Devices. 1st ed. New York: Marcel Dekker; 1997:11-21.

2. Interagency Registry for Mechanically Assisted Circulatory Support. INTERMACS manual of operations. Available at: http://www.uab.edu/ctsresearch/ intermacs/manuals.htm. Accessed May 10, 2011.

3. INTERMACS durable devices included in database. Available at: http://www. uab.edu/ctsresearch/intermacs/Document $\% 20$ Library/Appendix $\% 20 \mathrm{~K} \% 20 \%$ 20Device\%20Brand\%20List,\%20FINAL,\%2010-30-08.doc. Accessed May 10, 2011.

4. Altieri F, Berson A, Borovetz H, Butler K, Byrd G, Ciarkowski AA, et al. Longterm mechanical circulatory support system reliability recommendation: American Society for Artificial Internal Organs and Society of Thoracic Surgeons: long-term mechanical circulatory support system reliability recommendation ASAIO J. 1998;44:108-14.

5. Resnic FS, Normand ST. Postmarketing surveillance of medical devices-filling in the gaps. N Engl J Med. 2012;366:875-7.

6. Holman WL. Interagency Registry for Mechanically Assisted Circulatory Support (INTERMACS): what have we learned and what will we learn? Circulation. 2012;126:1401-6.

7. Kirklin JK. The importance of the mechanical circulatory support database: a plea for commitment. J Heart Lung Transplant. 2001;20:803-4.

8. Kirklin JK, Naftel DC, Kormos RL, Stevenson LW, Pagani FD, Miller MA, et al. Third INTERMACS Annual Report: the evolution of destination therapy in the United States. J Heart Lung Transplant. 2011;30:115-23.

9. Rose EA, Gelijns AC, Moskowitz AJ, Heitjan DF, Stevenson LW, Dembitsky WP, et al. Long-term use of a left ventricular assist device for endstage heart failure. $N$ Engl J Med. 2001;345:1435-43.

10. Rogers JG, Butler J, Lansman SL, Gass A, Portner PM, Pasque MK, et al. Chronic mechanical circulatory support for inotrope-dependent heart failure patients who are not transplant candidates: results of the INTrEPID Trial. J Am Coll Cardiol. 2007;50:741-7.

11. Mussivand T, Hetzer R, Vitali E, Meyns B, Noirhomme P, Koerfer R, et al. Clinical results with an ePTFE inflow conduit for mechanical circulatory support. J Heart Lung Transplant. 2004;23:1366-70.

12. Pagani FD, Long JW, Dembitsky WP, Joyce LD, Miller LW. Improved mechanical reliability of the HeartMate XVE left ventricular assist system. Ann Thorac Surg. 2006;82:1413-8.

13. Miller LW, Pagani FD, Russell SD, John R, Boyle AJ, Aaronson KD, et al. Use of a continuous-flow device in patients awaiting heart transplantation. $N$ Engl J Med. 2007;357:885-96.

14. Potapov EV, Ignatenko S, Nasseri BA, Loebe M, Harke C, Bettmann M, et al. Clinical significance of PIA polymorphism of platelet GP IIb/IIIa receptors during long-term VAD support. Ann Thorac Surg. 2004;77:869-74.

15. Boyle AJ, Russell SD, Teuteberg JJ, Slaughter MS, Moazami N, Pagani FD, et al Low thromboembolism and pump thrombosis with the HeartMate II left ventricular assist device: analysis of outpatient anti-coagulation. J Heart Lung Transplant. 2009;28:881-7.

16. Pamboukian SV, Tallaj JA, Brown RN, Holman WL, Blood M, George JF, et al Improvement in 2-year survival for ventricular assist device patients after implementation of an intensive surveillance protocol. $J$ Heart Lung Transplant. 2011; 30:879-87.

17. Slaughter MS, Rogers JG, Milano CA, Russell SD, Conte JV, Feldman D, et al Advanced heart failure treated with continuous-flow left ventricular assist device. N Engl J Med. 2009;361:2241-51.

18. Pagani FD, Miller LW, Russell SD, Aaronson KD, John R, Boyle AJ, et al. Extended mechanical circulatory support with a continuous-flow rotary left ventricular assist device. J Am Coll Cardiol. 2009;54:312-21.

19. Kirklin JK, Naftel DC, Pagani FD, Kormos RL, Stevenson L, Miller M, et al. Long-term mechanical circulatory support (destination therapy): on track to compete with heart transplantation? J Thorac Cardiovasc Surg. 2012;144: 584-603. 


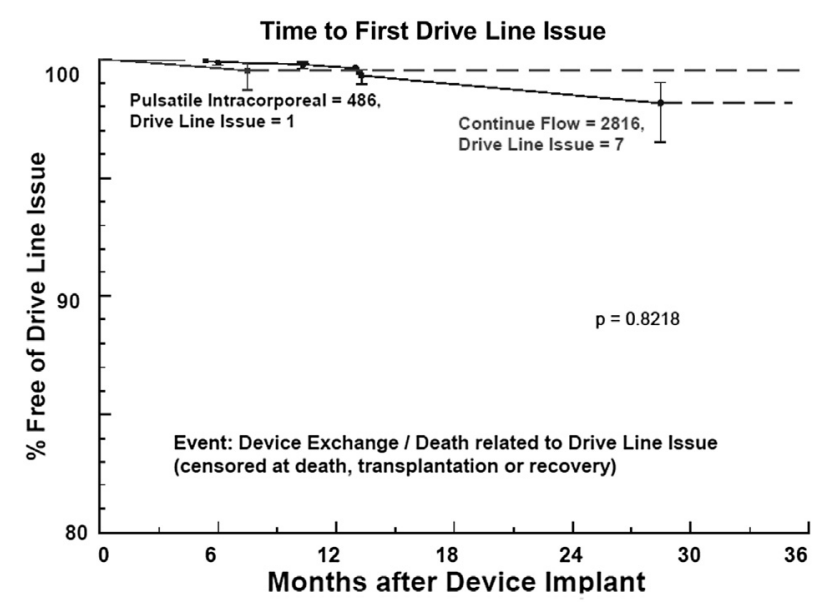

FIGURE E1. Deaths and device exchanges from driveline failure are shown for pulsatile and continuous flow ventricular assist devices $(P<.8218$; pulsatile vs continuous flow ventricular assist devices).
TABLE E1. Possible outcomes for patients supported with ventricular assist device and reasons for pump exchange or device-related death

\begin{tabular}{lcc}
\hline & \multicolumn{2}{c}{ Pump type } \\
\cline { 2 - 3 } \multicolumn{1}{c}{ Reason } & Continuous flow & $\begin{array}{c}\text { Pulsatile } \\
\text { intracorporeal flow }\end{array}$ \\
\hline Infection & $7(13.46)$ & $4(7.70)$ \\
Pump related & & \\
$\quad$ Thrombosis/hemolysis & $28(53.85)$ & $6(11.54)$ \\
Drive line & $7(13.46)$ & $1(1.92)$ \\
Drive unit & $10(19.23)$ & $41(78.85)$ \\
Total & $52(100)$ & $52(100)$ \\
\hline Data presented as n (\%). & &
\end{tabular}

Time to First Thrombosis/Hemolysis Issue

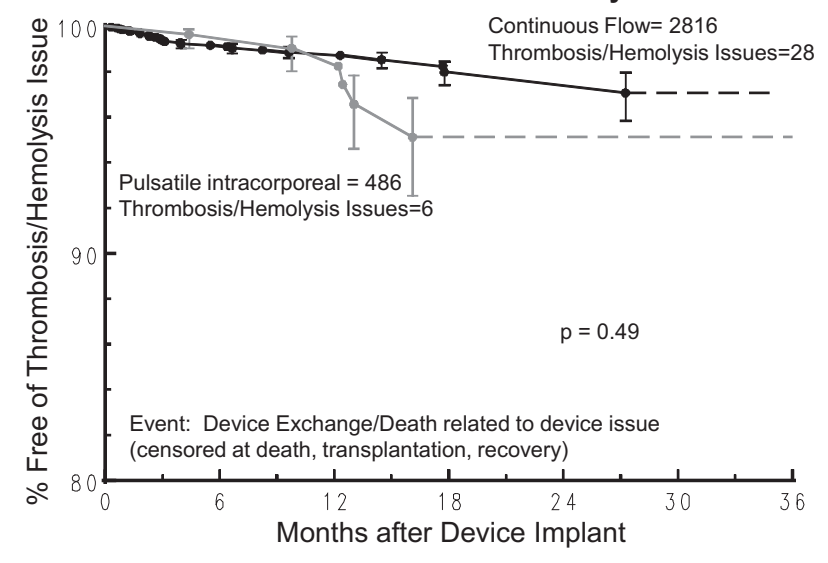

FIGURE E2. Deaths and device exchanges from thrombosis/hemolysis are shown for pulsatile and continuous flow ventricular assist devices $(P<.2501$; pulsatile vs continuous flow ventricular assist devices). 